\title{
Special issue: Advances in convection-permitting climate modeling
}

\author{
Andreas F. Prein ${ }^{1} \cdot$ Roy Rasmussen ${ }^{1} \cdot$ Christopher L. Castro ${ }^{2} \cdot$ Aiguo Dai $^{3} \cdot$ Justin Minder $^{3}$
}

Published online: 24 April 2020

(c) Springer-Verlag GmbH Germany, part of Springer Nature 2020

Convection-permitting or kilometer-scale climate models are modeling systems that allow the explicit simulation of atmospheric deep convection and a much-improved representation of orography and land-surface heterogeneities compared to traditional climate models with much coarser resolution. Convection-permitting models (CPMs) have been shown to result in a drastic improvement in simulating essential hydro-climatological processes such as convective and orographic precipitation, extreme rainfall events, soil-atmosphere feedbacks, cloud-radiation feedbacks, and mountain snowpack. This special issue of Climate Dynamics includes 31 papers that investigate a large variety of earth system processes over regions in Africa, Asia, Europe, and North America. The peer-reviewed articles show that CPM models consistently improve various long-standing issues in atmospheric modeling but also highlight remaining challenges and emerging research topics that can guide future research.

The aim of this special issue is to improve the coordination of CPM research within the climate modeling community and to enhance the communication of results to related fields such as weather forecasting, impact research, and stakeholder communities. The primary goal is to improve our understanding, through the added value of CPMs, of the past, current, and future climates in different regions, and to provide a different modeling system compared to coarser resolution state-of-the-art global climate models.

Papers in this special issue document tremendous progress in CPM modeling. This includes the first CPM climate simulations over continental-scales covering most of

Andreas F. Prein

prein@ucar.edu

1 National Center for Atmospheric Research (NCAR), 3090 Center Green Dr., Boulder, CO 80301, USA

2 University of Arizona, 1133 E. James E. Rogers Way, PO Box 210011, Tucson, AZ 85721-0011, USA

3 University at Albany, 1400 Washington Ave., Albany, NY 12222, USA
Europe and North America and the introduction of novel modeling systems that allow seamless downscaling of global simulations to kilometer-scales over target regions with nonhydrostatic variable resolution models. Additionally, the first CPM multi-model ensemble simulations that allow unprecedented intercomparison of modeling systems and insights into the robustness of CPM added values are presented. The articles in this special issue apply CPMs to a large variety of processes such as continental precipitation, tropical convection, monsoon circulations, atmospheric radiation, orographic processes, soil-atmosphere coupling, and extreme events. Promising results are shown for all of these topics highlighting the potential of CPMs to resolve long-standing issues in climate models.

Besides these encouraging results, several challenges in CPM climate modeling are identified that should guide future research. A key challenge is the lack of high-resolution observational datasets in many regions of the world making a process-based evaluation of CPM simulations difficult. Additional challenges arise due to the high computational demands of CPM climate simulations and their large data output volume. The high computational costs make it difficult to run CPM simulations in large ensembles, over long time periods, or large domains. Because of this, how to construct representative lateral forcing data for driving the CPMs becomes increasingly important. This also makes it hard to assess uncertainties in CPM simulations and to compare them to existing global climate model (GCMs) results. Furthermore, the large output data volume poses a challenge for data archiving, analysis, and sharing. Porting CPM code to run on modern computer architectures (e.g., graphical processor units) and moving towards online model evaluation are promising pathways for addressing these challenges. CPM models operate in the turbulent gray zone and some of their parameterizations (e.g., turbulence, radiation, land surface parameterizations) were developed for applications in much coarser resolution models, which can introduce systematic biases. Future research should focus on the development of scale aware parameterizations that can reliably operate at kilometer-scales. 
This special issue highlights the potential of CPMs to revolutionize climate modeling by using a more physically based approach. This will improve our understanding of climate change impacts on the earth system and will allow unprecedented insights into societal relevant processes such as changes in water availability and extreme events.
Publisher's Note Springer Nature remains neutral with regard to jurisdictional claims in published maps and institutional affiliations. 